\title{
Construcción de un instrumento de valoración de la expresividad musical en profesorado y alumnado en la Enseñanza Superior de Música
}

\section{Carolina Bonastre ${ }^{1}$, Roberto Nuevo ${ }^{2}$}

${ }^{1}$ Universidad Complutense de Madrid

${ }^{2}$ Universidad Rey Juan Carlos

\section{España}

Correspondencia: Carolina Bonastre. Universidad Complutense de Madrid. E-mail: cbonastr@ucm.es

(C) Universidad de Almería and Ilustre Colegio Oficial de la Psicología de Andalucía Oriental (Spain) 


\section{Resumen}

Introducción. Una de las principales funciones de la música es la expresión y comunicación de emociones. El aprendizaje de las habilidades centradas en esta comunicación es una parte central en la formación de los intérpretes y, el estudio de las concepciones de profesores y alumnos sobre los modos adecuados de mejorar la expresividad puede considerarse una de las tareas prioritarias para la investigación psicoeducativa. Por ello, este trabajo está dirigido a: a) validar un instrumento para su evaluación; b) conocer la valoración de diferentes estrategias de enseñanza-aprendizaje de la expresividad emocional en la música, en profesores y alumnos de niveles superiores de educación musical y c) comparar esas valoraciones en ambos grupos.

Método. Para ello, 229 personas (170 estudiantes y 59 profesores) del Conservatorio Superior de Música de Madrid completaron un cuestionario sobre la mejora de la expresividad con cuatro estrategias distintas de enseñanza-aprendizaje.

Resultados. Se encontraron algunas diferencias estadísticamente significativas entre grupos respecto a las preferencias de dichos modelos. Se pueden destacar las diferencias en intragrupo: la estrategia basada en el uso de instrucciones técnicas fue la mejor valorada mientras que el modelado recibió la peor valoración. Estos efectos fueron independientes del sexo y la edad. La estructura factorial teórica tuvo un excelente ajuste a los datos ( $C F I=.95)$.

Discusión y conclusiones. En conclusión, ambos grupos difieren en sus concepciones sobre las estrategias óptimas para trabajar la expresividad, pero coinciden en las preferencias sobre una u otra estrategia, valorando especialmente el uso de instrucciones técnicas. Se discuten las posibles implicaciones pedagógicas y se propone este cuestionario como herramienta para su uso en contextos educativos.

Palabras Clave: Aprendizaje musical, Educación Superior, Emoción, Expresión, Interpretación. 


\begin{abstract}
Introduction. Expressing and communicating emotions is one of the main functions of music. Focusing on such communication process is a core part of performer's education and studying conceptions of teachers and students about ways of improving expressivity seems a priority in psychoeducational research. Therefore, this work aimed to: a) validating a tool for assessing them; b) knowing valuations about different models for teaching and learning of emotional expressivity in music among Higher Education teachers and students of music; c) comparing those valuations between both groups.
\end{abstract}

Method. For this purpose, 229 persons (170 students and 59 teachers) from the Music Conservatory of Madrid completed a questionnaire about improving expressiveness. Four different strategies for teaching and learning expressiveness were included.

Results. There were certain statistically significant differences between groups in their preferences for different strategies. The most important differences, however, were found in within-group comparisons: the strategy based on the use of technical instructions received the best ratings, whereas modelling was the strategy with the poorest ratings. These effects were independent of gender and age. The theoretical factor structure had excellent fit to the data $(C F I=.95)$.

Discussion or conclusion. In conclusion, the two groups differed in their conceptions about the best strategies for training in expressiveness, but they agreed on their preferences for one strategy or another, with particular preference for the use of technical instructions. The possible pedagogical implications of these differences are discussed, and this questionnaire is recommended for use in educational settings.

Keywords: Learning music, Higher Education, Emotion, Expression, Performance. 


\section{Introducción}

La interpretación musical es principalmente, aunque no exclusivamente, un medio de expresión y comunicación de emociones, lo que puede reflejarse tanto en emociones básicas (Gabrielsson y Juslin, 2003) como en la evocación de emociones mediante medios estéticos (Doğantan-Dack, 2014). Además, la comunicación emocional es valorada visiblemente en los diferentes niveles de aprendizaje (Juslin y Laukka, 2004). La expresividad emocional depende en gran medida de las destrezas musicales del intérprete, por lo que es importante conocer las herramientas expresivas que permiten transmitir emociones al oyente. Actualmente hay amplia evidencia de que las destrezas expresivas pueden ser enseñadas y aprendidas con eficacia y que el desarrollo de las habilidades musicales supone un incremento progresivo en el manejo consciente de elementos técnicos y estilísticos para conseguir efectos expresivos concretos (Meissner, 2017; Meissner y Timmers, 2019; Viellard, Roy y Peretz, 2012). Del mismo modo, oyentes (Juslin y Laukka, 2004) y músicos (Lindström, Juslin, Bresin y Williamon, 2003) destacan la importancia de la necesidad de una comunicación expresiva.

En otras disciplinas afines, como es el caso de la expresión corporal, algunos estudios recientes también sugieren que en España se recibe una atención insuficiente en la formación de los futuros docentes (Sánchez y López, 2019). Esto es destacable ya que la expresión musical y su aprendizaje depende también en gran medida del movimiento y la expresión corporal del intérprete (Massie-Laberge, Cosette y Wanderley, 2020). Así, pese a que se aprecia una intención en el ámbito de la enseñanza musical hacia un cambio de paradigma desde un enfoque en la acumulación de conocimientos y el virtuosismo hacia posiciones constructivistas (Torrado y Pozo, 2008), parece que la mayoría de los profesores de música siguen apegados a una enseñanza tradicional centrada en adquirir resultados supervisados por el profesor (Bautista y Pérez Echevarría, 2008; López Íñiguez, Pozo y de Dios, 2014), sin que el mensaje constructivista explícito haya supuesto un cambio efectivo en el aula (Garnett, 2013; Karlsson y Juslin, 2008).

En esta línea, algunos estudios dirigidos a la realidad del aula sugieren que las capacidades expresivas tienden a no trabajarse, en general, explícitamente en la práctica, ni con tareas determinadas ni mediante indicaciones curriculares específicas (Karlsson y Juslin, 2008; Young, Burwell, y Pickup, 2003). En algunos casos, tan solo se comentan aspectos 
relacionados con el fin de alcanzar una comprensión artística general de la pieza (Chaffin y Lemieux, 2005). Por tanto, el alumno puede no recibir retroalimentación correctiva sobre su ejecución expresiva.

Aunque la investigación en este campo es todavía escasa, se han llevado a cabo diferentes propuestas para la mejora de habilidades expresivas en músicos como la evaluación de un entrenamiento específico en estudiantes de música consiguiendo mayor implicación emocional con la pieza, mayor comprensión estructural y mejora en la ejecución expresiva (Sloboda, Gayford y Minassian, 2003); el uso de retroalimentación cognitiva en músicos durante sus actuaciones con mejoras significativas en la comunicación emocional (Juslin, Karlsson, Lindström, Friberg y Schoonderwaldt, 2006); estrategias dialógicas, basadas más en el diálogo que en las instrucciones unidireccionales del profesor (Meissner y Timmers, 2019; Meissner y Timmers, 2020); o el uso de distintos sistemas de representación para reconstruir la propia experiencia musical (Pozo, Torrado y Pérez-Echeverría, 2019).

En esta línea, Woody (2006) comparó los resultados de tres estrategias para el aprendizaje de la expresividad y encontró que la eficacia era similar aunque: a) la imitación o modelado suponía mejoras pequeñas pero consistentes; b) el uso de metáforas e imágenes se relacionó con cambios más largos pero no siempre en la dirección esperada; c) dar instrucciones específicas parecía aportar más beneficios para personas con niveles más avanzados.

Por otra parte, algunos autores indican que la principal estrategia instruccional en la clase de música es el discurso del profesor (Young et al., 2003). En todo caso, y aunque la generalizabilidad del resultado de ese estudio pueda ser discutible, es relevante que el uso de una u otra estrategia específica parece estar muy influido por sus opiniones respecto a la naturaleza de la expresividad en la música. (Brenner y Strand, 2013). Una de esas creencias es que las instrucciones verbales sobre factores específicos dan un resultado no natural o una expresividad simulada (Williamon, 2014), lo que podría llevar a algunos profesores a evitar enseñarla o mejorarla en aquellos alumnos que creen que ya tienen ese potencial natural, para eludir afectarlo. Aunque la evidencia empírica indica que estas ideas u otras similares son inadecuadas, estas tienden a reafirmarse y ser muy resistentes al cambio y podrían tener un impacto directo en la práctica cotidiana en el aula (Juslin y Persson, 2002). 
En suma, aunque distintos autores reconocidos y relevantes han indicado desde hace décadas que la educación musical no debería estar exclusivamente enfocada en la técnica, debido al riesgo de obtener músicos sin expresividad (p.ej., Marchand, 1975), sólo recientemente se está tomando seriamente en consideración la necesidad de incluir explícitamente elementos de la expresión musical en la educación musical formal. La aproximación tradicional para hacer esto ha implicado cuatro estrategias básicas, de acuerdo con Juslin et al. (2006):

1) Modelado musical: el profesor hace de modelo y se asume que el estudiante va a aprender a través de la imitación.

2) Metáforas o imágenes: el profesor da instrucciones al alumno, como, por ejemplo, "ahora todo se pone oscuro" o "esta melodía fluye como el agua de un arroyo" haciendo referencia a representación de imágenes.

3) Hacer que el estudiante se centre en sus propias emociones: dar pautas que ayuden al alumno a enfocar su atención sobre cómo se siente mientras interpreta un texto musical, con la asunción de que las emociones activadas se transferirán a los resultados sonoros y facilitarán la expresión emocional adecuada.

4) Centrarse en aspectos técnicos de la ejecución: proporcionar directrices específicas para cambiar aspectos del sonido y conseguir un sentido más expresivo.

De todos modos, la distinción entre diferentes estrategias en la práctica real en el aula es algo arbitraria y un profesor idealmente usará diferentes estrategias individualmente o de forma combinada (Meissner, 2017) en función de las necesidades específicas de cada alumno, sus características y circunstancias personales y los objetivos buscados en cada momento. Pero para poder llevar a término esta acción es relevante ser conscientes de dichas estrategias y teniendo en cuenta los objetivos pedagógicos planteados por el docente para la mejora expresiva del alumno, distinguirlas y hacer un uso adecuado de ellas.

\section{Objetivos e hipótesis}

Este trabajo parte de la hipótesis de que los participantes tienen preferencia por alguna estrategia en particular y de que el instrumento elaborado es válido. Inicialmente, respecto al 
instrumento de medida se considera que cada una de las estrategias se diferenciará factorialmente de las demás y que los factores latentes presentarán covariaciones significativas entre ellos. También se plantea que los dos grupos incluidos, alumnos y profesores, dada la diferente instrucción musical recibida (Bautista y Pérez-Echevarría, 2008) podrían diferenciarse en las opiniones sobre cada una de estas cuatro estrategias. Además, se hipotetiza que las opiniones dentro de cada uno de los grupos diferirán respecto a las estrategias propuestas.

De acuerdo con Susan Hallam (2010) o Casas-Mas, Pozo y Montero (2014), estudiar las concepciones y valoraciones de estudiantes y docentes debería ser una meta primordial para la investigación en educación musical teniendo en cuenta que potencialmente tienen un papel moderador relevante en su enseñanza y aprendizaje (Bonastre, Muñoz y Timmers, 2017; González Rollo y Bautista, 2018). Por ello, los principales objetivos de este trabajo son: conocer las opiniones sobre estas estrategias de enseñanza-aprendizaje de la expresividad en la música; desarrollar y validar un instrumento para alcanzar este objetivo; y, por último, descubrir si existen diferencias en las concepciones entre los participantes, estudiantes y profesores de niveles superiores de educación musical.

\section{Método}

\section{Participantes}

La muestra estuvo compuesta por profesores y estudiantes del Real Conservatorio Superior de Música de Madrid (RCSMM), España. 229 participantes completaron los cuestionarios: 170 estudiantes, de los que un 49.7\% eran mujeres (edad media=21.5, desviación típica $(\mathrm{DT})=3.5$; rango:17-35) y el 50.3\% hombres (edad media=21.4, DT=3.1; rango:18-30); y 59 profesores, con un 59.3\% de mujeres (edad media=40.1; DT=8.3; rango: 23-56) y un 40.7\% de hombres (edad media=43.0, DT=11.6; rango:23-67).

El muestreo fue incidental: se trató de acceder a la mayor cantidad posible de estudiantes y profesores que aceptaran participar. Dados los objetivos principales del trabajo no se fijó un tamaño muestral mínimo, aunque para el análisis a realizar más exigente desde el punto de vista de la potencia estadística (un Análisis Factorial Confirmatorio), se considera necesario un grado de sobredeterminación que implique un mínimo de entre 5 y 10 personas 
por variable incluida (MacCallum, Widaman, Zhang y Hong, 1999); ya que en ese análisis se incluyen 16 ítems, un mínimo de entre 80 y 160 personas serían convenientes para que los análisis fueran fiables. La muestra obtenida supone un porcentaje importante de los alumnos y docentes del centro, aparte de cumplir los criterios estadísticos mínimos. Por otra parte, se puede asumir que el RCSMM es un centro que recoge alumnos de toda España, por lo que, con las debidas cautelas, la muestra puede permitir obtener resultados generalizables en dicho país en la educación superior musical. ${ }^{1}$

\section{Instrumentos}

Se elaboró un cuestionario con el fin de evaluar diferentes facetas de cada una de las cuatro estrategias de enseñanza-aprendizaje de la expresividad en la música previamente descritas, basándose en trabajos previos respecto a las estrategias reales usadas en el aula (p.ej., Juslin et al., 2006). Una versión inicial de cuestionario fue aplicada a un pequeño grupo de estudiantes (10) y a partir de los comentarios sobre la comprensión de las preguntas y las viñetas se modificó ligeramente para replantearlas. El cuestionario fue entonces evaluado por cuatro expertos externos y se aplicaron pequeñas correcciones de acuerdo a sus sugerencias, con lo que se obtuvo el cuestionario definitivo. Se compone de 20 ítems tipo Likert con cuatro categorías posibles de respuesta (de 1," nada" a 4, "mucho"), reflejando el grado de acuerdo con cinco afirmaciones para cada subescala relativas a un ejemplo específico de una situación de enseñanza y el tipo de estrategia usada respecto a la expresividad. Estos cinco ítems incluían cuatro aspectos: a) si como docente enseñaría la expresividad del modo reflejado en el ejemplo; b) si cree que es un método correcto; c) si cree que así el alumno aprenderá correctamente; y d) si cree que es el mejor modo de mejorar la expresividad. Se incluyó, además, una cuestión general referida a si alguna vez se había encontrado en las clases, como alumno o profesor, en una situación como la descrita en el ejemplo (los ítems específicos se detallan posteriormente en las tablas incluidas en la sección de Resultados). Esta pregunta adicional se incluyó en los análisis descriptivos de los ítems individuales y tiene, además de una función descriptiva, el rol potencial de observar relaciones entre las concepciones sobre la expresividad y la experiencia personal de los participantes. Sin embargo, no fue incluida para calcular la puntuación total de cada subescala ni en el resto de análisis estadísticos ya que la

${ }^{1}$ A falta de datos oficiales publicados, pueden recogerse las palabras genéricas de la directora del centro en su mensaje de bienvenida en la página WEB: "El Real Conservatorio recibe alumnos de todas las comunidades autónomas y de distintos continentes" (https://rcsmm.eu/el-centro/?m=1). 
respuesta no depende de las concepciones individuales. Por lo tanto, las puntuaciones totales para cada estrategia se construyeron sumando las puntuaciones en cada uno de los otros cuatro ítems: rango de 4 a 16.

Cada una de las situaciones representativas de cada estrategia se presentó en formato de viñetas narrativas, como una descripción detallada de un ejemplo en el que el profesor actúa de un modo acorde con cada estrategia. Los ejemplos concretos que se utilizaron se encuentran disponibles en otro trabajo (Bonastre y Timmers, 2019) y se pueden solicitar al autor principal del presente estudio. En la figura 1 se puede observar un ejemplo para el caso del modelado. El uso de viñetas tiene la ventaja de concretar lo que se quiere decir al hablar de una cierta circunstancia evitando ambigüedades. Este método ha sido ampliamente usado en investigación educativa (p.ej., Hughes y Huby, 2004).

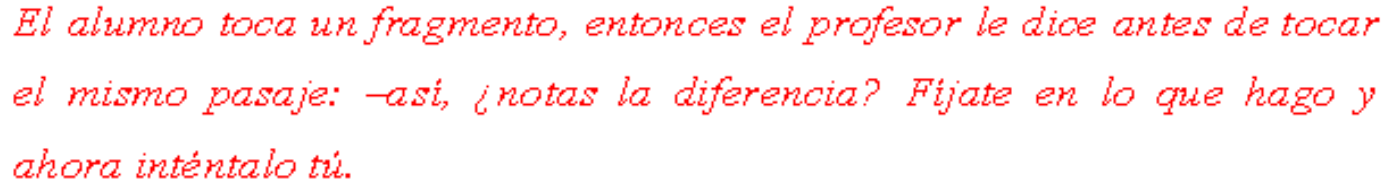

Figura 1. Ejemplo de viñeta describiendo una situación de enseñanza de la expresividad, referido al modelado.

\section{Procedimiento}

Se recopiló la información mediante la distribución de cuestionarios a alumnos y profesores del RCSMM. Se solicitó su participación y colaboración en esta investigación y se explicó brevemente los objetivos de la misma obteniendo un consentimiento informado verbal vinculado a completar y devolver voluntariamente los cuestionarios. Todos los participantes fueron informados de que su participación era totalmente libre y de que se respetaría el anonimato de sus respuestas.

\section{Análisis de datos}

En primer lugar, se analizó la estructura factorial del cuestionario mediante Análisis Factorial Confirmatorio (AFC). El modelo que se introdujo en el análisis (ver Figura 1, presentada más adelante) incluía cuatro factores latentes, uno por cada estrategia, asociados cada uno a las cuatro variables observadas referidas a esa estrategia; las covarianzas entre 
todos los factores fueron estimadas libremente. Para obtener la identificación estadística el peso factorial del primer ítem de cada factor fue fijado a 1, las correlaciones entre los errores de medida de cada variable observada fueron fijadas a 0 y los coeficientes de los errores sobre los ítems fueron fijados a 1. El método de estimación fue $A D F$ (del inglés Asymptotically Distribution Free), dada su potencial robustez ante la posible falta de normalidad multivariada y su adecuación para el análisis de datos categóricos.

De acuerdo con las recomendaciones usuales, se consideraron diferentes tipos de índices de ajuste del modelo, con los siguientes valores de referencia: chi-cuadrado no significativa; chi-cuadrado/Grados de Libertad $(C M I N / D F)<5 ; C F I>.90 ;$ GFI>.90; y RMSEA<.06 (Bentler, 1990). Se estimó la consistencia interna de cada subescala y de una posible escala total mediante el coeficiente alfa de Cronbach. Aunque los criterios de valoración de este coeficiente son de nuevo arbitrarios, suele aceptarse que valores superiores a .80 son buenos y por encima de .90 excelentes (p.ej., De Vellis, 2003; Lance, Butts y Michels, 2006).

A continuación, se realizaron análisis descriptivos (media y desviación típica) de todos los ítems (incluyendo el ítem que no se considera en el cálculo de las puntuaciones totales) y de las puntuaciones totales en cada grupo (alumnos y profesores) y las medias fueron comparadas mediante pruebas $\mathrm{T}$ de comparación de medias para muestras independientes. Adicionalmente, se estimaron tamaños del efecto ( $g$ de Hedges) para cada comparación; $g$ es una versión insesgada del popular estadístico $d$ de Cohen y pueden asumirse los criterios usuales para interpretar los valores de $d$ (Cohen, 1988): por debajo de 0.20 es un efecto irrelevante, entre 0.20 y 0.50 un efecto pequeño, entre 0.50 y 0.80 un efecto medio y por encima de 0.80 un efecto elevado. Por supuesto, estos criterios son arbitrarios y la relevancia de un efecto depende del campo de estudio específico. Dado que las variables dependientes (VDs) en el caso de los contrastes sobre los ítems son categóricas y con un rango de puntuación limitado (1-4), se realizaron además contrastes no paramétricos (W de Wilcoxon), que no impone asunciones de normalidad y simetría como las pruebas $\mathrm{T}$, y en caso de discrepancia con el resultado de los contrastes de medias esta diferencia en los resultados se indicará específicamente. Se realizaron contrastes de normalidad (Shapiro-Francia) para las medidas totales sobre la valoración de las cuatro estrategias de enseñanza-aprendizaje de la expresividad: un valor de $p>.05$ permite asumir que la distribución de las puntuaciones es normal. 
Se analizaron las diferencias intra-grupo entre cada una de las susbescalas, tanto para la muestra total como separadamente por grupos, mediante pruebas $\mathrm{T}$ de contraste de medias para muestras relacionadas. En este caso, lo que se comparan no son diferencias entre grupos, sino diferencias entre variables dentro del mismo grupo. Igualmente, se compararon mediante pruebas T para muestras relacionadas las puntuaciones, separadamente por grupos, entre la frecuencia informada de uso de cada una de las estrategias, es decir, con qué frecuencia decían haber estado alguna vez en clase en cada una de las situaciones descritas (la pregunta no incluida en el cálculo de las puntuaciones totales de cada subescala).

Finalmente, con el doble objetivo de comprobar las diferencias entre grupos bajo condiciones más restrictivas (controlando estadísticamente por el sexo) y de reducir el posible sesgo de cometer errores tipo I debido a comparaciones múltiples, se realizó un análisis de regresión lineal multivariada en el que las puntuaciones en los cuatro casos de enseñanzaaprendizaje de la expresividad emocional ejemplificados fueron incluidas como variables dependientes (VDs), el grupo como variable independiente (VI) y el sexo como covariable (hombre $=0$, considerado como categoría de referencia). Tras la prueba ómnibus se analizaron los efectos específicos de cada estrategia, así como la posible presencia de interacciones entre grupo y sexo. En el análisis se incluyó la estimación de una constante de la función.

Todos los análisis estadísticos se realizaron con el software STATA para Windows, versión 13.1 y MPlus 6.0.

\section{Resultados}

En primer lugar, respecto al Análisis Factorial Confirmatorio para comprobar la adecuación de la estructura del cuestionario se encontró un ajuste adecuado del modelo total (Chi-square $(98)=238.9, p<.001 ;$ CMIN/ DF $=2.44 ; C F I=.948 ; T L I=.936 ; R M S E A$ $=.082(90 \%$ CI: .069, .096). Todos los pesos factoriales fueron elevados y estadísticamente significativos $(p<.001)$ y no se encontró ningún signo de cargas cruzadas entre los resultados. Las covarianzas estimadas entre las diferentes estrategias fueron muy reducidas salvo entre metáforas y emociones (covarianza=0.60). La consistencia interna (coeficiente alfa de Cronbach) fue elevada para las diferentes subescalas y para la escala total (superiores a .90 en todos los casos). En la Tabla 3 se muestran los coeficientes no estandarizados para cada ítem 
en su modelo teórico. En la Figura 2 se presenta la estructura del modelo que se analizó y se representan los coeficientes estandarizados.

Tabla 1. Resultados del Análisis Factorial Confirmatorio de la escala de medida sobre la enseñanza de la expresividad en la música.

\begin{tabular}{|c|c|c|}
\hline Variables & $\begin{array}{c}\text { Pesos no } \\
\text { estandarizados (ET) }\end{array}$ & $\begin{array}{c}\text { Intervalo de } \\
\text { Confianza }(95 \%) \\
\end{array}$ \\
\hline \multicolumn{3}{|l|}{ MODELADO } \\
\hline $\begin{array}{l}\text { ¿Si tuvieras que dar clases crees que lo } \\
\text { harías así? }\end{array}$ & 1.0 & --- \\
\hline ¿Piensas que este método es correcto? & $1.05(0.06)$ & 0.94 .1 .17 \\
\hline $\begin{array}{l}\text { ¿Piensas que el estudiante aprenderá } \\
\text { correctamente? }\end{array}$ & $0.97(0.06)$ & 0.86 .1 .08 \\
\hline $\begin{array}{l}\text { ¿Crees que es el mejor modo para mejorar } \\
\text { la expresividad? }\end{array}$ & $0.89(0.06)$ & 0.76 .1 .01 \\
\hline \multicolumn{3}{|l|}{ METAFORAS O IMAGENES } \\
\hline $\begin{array}{l}\text { ¿Si tuvieras que dar clases crees que lo } \\
\text { harías así? }\end{array}$ & 1.0 & --- \\
\hline ¿Piensas que este método es correcto? & $0.97(0.06)$ & 0.85 .1 .10 \\
\hline $\begin{array}{l}\text { ¿Piensas que el estudiante aprenderá } \\
\text { correctamente? }\end{array}$ & $0.99(0.06)$ & 0.86 .1 .11 \\
\hline $\begin{array}{l}\text { ¿Crees que es el mejor modo para mejorar } \\
\text { la expresividad? }\end{array}$ & $0.94(0.07)$ & 0.79 .1 .08 \\
\hline
\end{tabular}

USO DE LAS PROPIAS EMOCIONES

¿Si tuvieras que dar clases crees que lo harías así?

1.0

¿Piensas que este método es correcto?

$0.99(0.06) \quad 0.88 .1 .11$

¿Piensas que el estudiante aprenderá

$1.04(0.06)$

0.94 .1 .16

correctamente?

$0.98(0.06)$

0.86. 1.10

¿Crees que es el mejor modo para mejorar la expresividad?

\section{TÉCNICA}

¿Si tuvieras que dar clases crees que lo harías así?

¿Piensas que este método es correcto?

1.0

$1.16(0.08)$

1.01. 1.32

¿Piensas que el estudiante aprenderá correctamente?

$1.09(0.08)$

0.94. 1.24

$¿$ Crees que es el mejor modo para mejorar la expresividad?

$1.08(0.08)$

0.92 .1 .25

- ET: error típico.

-Todos los pesos fueron estadísticamente significativos con $p<.001$. 


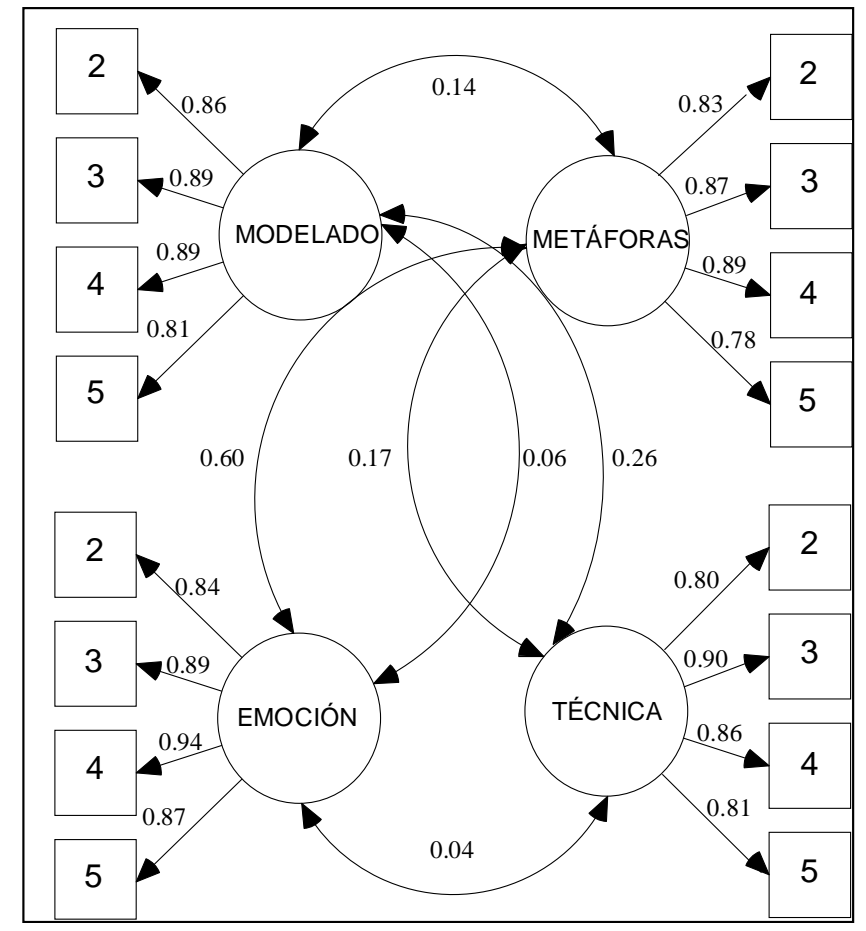

Figura 2. Representación gráfica de la estructura factorial del modelo de estrategias analizado con los pesos estandarizados entre cada factor latente y los ítems.

En las comparaciones entre profesores y estudiantes para las puntuaciones totales en cada una de las cuatro estrategias planteadas de enseñanza-aprendizaje de la expresividad, solo hubo diferencias estadísticamente significativas para el uso de metáforas o imágenes $(t(223)=2.46 ; p=.015)$ con una puntuación mayor para los alumnos, aunque el tamaño del efecto fue reducido $(g=0.37)$. Las pruebas de normalidad para estas puntuaciones totales (Shapiro-Francia), permitieron asumir la normalidad de las puntuaciones ( $p>.05$ ) y usar las puntuaciones totales en el resto de análisis como normales. En las comparaciones entre grupos para ítems específicos, sólo se encontraron diferencias en dos ítems, ambos referidos al uso de metáforas o imágenes y con puntuaciones superiores para los alumnos en los dos casos y tamaños del efecto moderados: si cree adecuado que el profesor haga esto $(t(224)=2.44$; $p=.015)$ y si consideran que es la mejor estrategia $(t(225)=2.50 ; p=.013)$. Estos resultados con datos descriptivos para las escalas y los ítems específicos se presentan con detalle en la Tabla 1. Además, según los contrastes no paramétricos, en otros dos ítems, también referidos a metáforas o imágenes se encontraron diferencias estadísticamente significativas que no aparecían en los contrastes paramétricos: si creen que lo harían como profesores $(z=2.08$; 
$p=.038)$ y si creen que el alumno aprenderá correctamente con este método $(z=2.46 ; p=.014)$, con rangos de puntuación superiores para los alumnos. No hubo otras discrepancias entre pruebas paramétricas y no paramétricas.

Tabla 2. Datos descriptivos y comparación entre alumnos y profesores.

\begin{tabular}{|c|c|c|c|c|}
\hline & Estudiantes & Profesores & $t(p) *$ & $g^{*}$ \\
\hline & Media (DT) & Media (DT) & $t(p)$ & \\
\hline Modelado & $8.81(3.21)$ & $8.93(3.26)$ & $0.25(.807)$ & 0.04 \\
\hline ¿Has estado alguna vez en una situación así? & $2.96(0.86)$ & $2.88(1.00)$ & $0.6(.543)$ & 0.09 \\
\hline $\begin{array}{l}\text { ¿Si tuvieras que dar clases crees que lo harías } \\
\text { de ese modo? }\end{array}$ & $2.22(0.92)$ & $2.29(0.95)$ & $0.5(.615)$ & 0.08 \\
\hline ¿Piensas que este método es correcto? & $2.35(0.96)$ & $2.31(0.91)$ & $0.3(.739)$ & 0.05 \\
\hline $\begin{array}{l}\text { ¿Piensas que el estudiante aprenderá } \\
\text { correctamente? }\end{array}$ & $2.25(0.85)$ & $2.25(0.88)$ & $0.0(.999)$ & 0.00 \\
\hline $\begin{array}{l}\text { ¿Crees que es el mejor modo para mejorar la } \\
\text { expresividad? }\end{array}$ & $1.99(0.88)$ & $2.12(0.85)$ & $1.0(.313)$ & 0.15 \\
\hline Metáforas o imágenes & $11.44(2.66)$ & $10.40(3.07)$ & $2.5(.015)$ & 0.37 \\
\hline ¿Has estado alguna vez en una situación así? & $2.75(0.96)$ & $2.68(0.95)$ & $0.5(.642)$ & 0.07 \\
\hline $\begin{array}{l}\text { ¿Si tuvieras que dar clases crees que lo harías } \\
\text { de ese modo? }\end{array}$ & $2.78(0.81)$ & $2.54(0.88)$ & $1.9(.057)$ & 0.29 \\
\hline ¿Piensas que este método es correcto? & $3.03(0.76)$ & $2.75(0.80)$ & $2.4(.015)$ & 0.37 \\
\hline $\begin{array}{l}\text { ¿Piensas que el estudiante aprenderá } \\
\text { correctamente? }\end{array}$ & $2.89(0.71)$ & $2.71(0.85)$ & $1.6(.123)$ & 0.23 \\
\hline $\begin{array}{l}\text { ¿Crees que es el mejor modo para mejorar } \\
\text { la expresividad? }\end{array}$ & $2.72(0.81)$ & $2.41(0.82)$ & $2.5(.013)$ & 0.38 \\
\hline Uso de las propias Emociones & $10.66(3.13)$ & $10.32(3.13)$ & $0.7(.488)$ & 0.11 \\
\hline ¿Has estado alguna vez en una situación así? & $2.60(0.97)$ & $2.62(1.01)$ & $0.21(.834$ & 0.03 \\
\hline $\begin{array}{l}\text { ¿Si tuvieras que dar clases crees que lo harías } \\
\text { de ese modo? }\end{array}$ & $2.67(0.92)$ & $2.55(0.80)$ & $0.9(.373)$ & 0.14 \\
\hline ¿Piensas que este método es correcto? & $2.78(0.84)$ & $2.66(0.86)$ & $1.0(.334)$ & 0.15 \\
\hline $\begin{array}{l}\text { ¿Piensas que el estudiante aprenderá } \\
\text { correctamente? }\end{array}$ & $2.66(0.86)$ & $2.66(0.83)$ & $0.0(.967)$ & 0.01 \\
\hline $\begin{array}{l}\text { ¿Crees que es el mejor modo para mejorar la } \\
\text { expresividad? }\end{array}$ & $2.53(0.84)$ & $2.45(0.89)$ & $0.6(.525)$ & 0.10 \\
\hline Técnica & $11.96(2.72)$ & $12.23(2.78)$ & $0.6(.529)$ & 0.10 \\
\hline ¿Has estado alguna vez en una situación así? & $3.20(0.84)$ & $3.30(0.82)$ & $0.8(.435)$ & 0.12 \\
\hline $\begin{array}{l}\text { ¿Si tuvieras que dar clases crees que lo harías } \\
\text { de ese modo? }\end{array}$ & $3.04(0.76)$ & $3.14(0.72)$ & $0.9(.395)$ & 0.13 \\
\hline ¿Piensas que este método es correcto? & $3.12(0.77)$ & $3.14(0.77)$ & $0.2(.831)$ & 0.03 \\
\hline $\begin{array}{l}\text { ¿Piensas que el estudiante aprenderá } \\
\text { correctamente? }\end{array}$ & $2.99(0.73)$ & $3.09(0.85)$ & $0.8(.425)$ & 0.12 \\
\hline $\begin{array}{l}\text { ¿Crees que es el mejor modo para mejorar la } \\
\text { expresividad? }\end{array}$ & $2.82(0.83)$ & $2.86(0.77)$ & $0.3(.740)$ & 0.05 \\
\hline
\end{tabular}

Nota. DT: desviación típica; $t: t$ de Student con grados de libertad=N-2; $g$ : tamaño del efecto $g$ de Hedges.; Resaltadas en negrita las comparaciones con diferencias estadísticamente significativas ( $\mathrm{p}<.05)$; * Los valores de $t$ y $g$ se expresan en valores absolutos. Los contrastes son bilaterales y la dirección de las diferencias se interpreta en función de los datos descriptivos.

En las comparaciones intra-grupo, se encontró un patrón similar tanto para el total de la muestra, como para las comparaciones realizadas por separado entre cada uno de los grupos: 
la estrategia basada en el uso de la técnica fue la mejor valorada, con diferencias estadísticamente significativas respecto a las otras tres, salvo en el caso de los alumnos donde no se encontraron diferencias entre técnica y el uso de metáforas o imágenes; la basada en el uso de metáforas o imágenes fue significativamente mejor valorado que los otros dos (excepto en el caso de los profesores, donde no se encontraron diferencias entre esta estrategia y la basada en el uso de las propias emociones); y la basada en el uso de las propias emociones fue en todos los casos mejor valorada que el modelado. Los tamaños del efecto fueron en general medio-altos para las comparaciones entre técnica y el resto de las estrategias y entre todas y el modelado. Se presenta un resumen de los resultados de todas las comparaciones en la Tabla 2.

Las comparaciones intra-grupo sobre la frecuencia de haber vivido en clase las situaciones descritas en cada caso, por otra parte, mostraron que para ambos grupos el uso de la técnica era la situación más frecuente con diferencias estadísticamente significativas respecto a las otros tres estrategias $(p<.001$ en todos los casos, con tamaños del efecto elevados o moderados - $g>0.50$, máximo de 0.88 -, salvo en la comparación con modelado en el caso de los alumnos: $g=0.27)$. El modelado, por su parte, fue más frecuente en los alumnos que el uso de metáforas $(p=.029 ; g=0.22)$ y que el uso de emociones $(p<.001 ; g=0.41)$. En el caso de los profesores, de modo similar, el modelado fue más frecuentemente usado que metáforas $(p=.047 ; g=0.36$ ), aunque no para el caso de emociones $(p=.077 ; g=0.26)$. Por otra parte, metáforas fue más frecuente que emociones solo entre los alumnos, aunque con un efecto pequeño $(p=.031 ; g=0.21)$, pero no entre los profesores $(p=.582 ; g=0.08)$.

Finalmente, los resultados del análisis de regresión múltiple, con la puntuación en las diferentes estrategias como VDs, el grupo como VI y sexo y edad como covariables, subrayan el mismo patrón de resultados de las comparaciones univariadas: en los contrastes ómnibus, solo para la puntuación en metáforas se encontró un efecto estadísticamente significativo $(F(2,209)=4.43 ; p=.013)$. En las comparaciones específicas para cada una de las estrategias solo el grupo, pero no el sexo, tuvo un efecto estadísticamente significativo en la basada en las metáforas, asociada a una mayor puntuación para los alumnos (coeficiente: 1.22; IC 95\%: $0.35,2.08 ; t(209)=2.78, p=.006)$. 
Tabla 3. Resultados de las comparaciones entre cada una de las estrategias de enseñanzaaprendizaje de la expresividad, para cada uno de los grupos y para la muestra

\begin{tabular}{lcccccc}
\hline Estrategias & \multicolumn{2}{c}{ Metáforas } & \multicolumn{2}{c}{ Emociones } & \multicolumn{2}{c}{ Técnica } \\
\cline { 2 - 7 } & $\mathrm{t}(\mathrm{p})^{*}$ & $\mathrm{~g}(\mathrm{IC} 95 \%)^{*}$ & $\mathrm{t}(\mathrm{p})$ & $\mathrm{g}(\mathrm{IC} 95 \%)$ & $\mathrm{t}(\mathrm{p})$ & $\mathrm{g}(\mathrm{IC} 95 \%)$ \\
\hline Alumnos & & & & & & \\
Modelado & $9.2(<.001)$ & $0.88(0.66 .1 .11)$ & $5.4(\mathrm{p}<.001)$ & $0.58(0.36 .0 .80)$ & $10.5(<.001)$ & $1.06(0.83 .1 .29)$ \\
Metáforas & --- & --- & $-3.6(.001)$ & $-0.27(-0.05 .-0.48)$ & $1.6(.102)$ & $0.20(-0.02 .0 .41)$ \\
Emociones & --- & --- & --- & --- & $4.0(<.001)$ & $0.44(0.23 .0 .66)$ \\
Profesores & & & & & & \\
Modelado & $2.45(.018)$ & $0.46(0.09 .0 .83)$ & $2.53(.014)$ & $0.43(0.06 .0 .80)$ & $6.9(<.001)$ & $1.08(0.69 .1 .47)$ \\
Metáforas & --- & --- & $-0.1(.923)$ & $-0.02(-0.35 .0 .39)$ & $3.7(.001)$ & $0.62(0.25 .0 .99)$ \\
Emociones & --- & -- & --- & --- & $3.5(.001)$ & $0.64(0.26 .1 .01)$ \\
Total & & & & & & \\
Modelado & $8.8(<.001)$ & $0.77(0.58 .0 .96)$ & $5.9(<.001)$ & $0.55(0.36 .0 .73)$ & $12.6(<.001)$ & $1.07(0.87 .1 .27)$ \\
Metáforas & --- & --- & $-3.2(.002)$ & $-0.20(-0.39 .-0.01)$ & $3.3(.001)$ & $0.31(0.12 .0 .50)$ \\
Emociones & --- & --- & --- & --- & $5.2(<.001)$ & $0.50(0.31 .0 .69)$ \\
\hline
\end{tabular}

* t: t de Student con grados de libertad=N-1; g: tamaño del efecto g de Hedges en valor absoluto; IC: Intervalo de Confianza al 95\%. Los valores descriptivos relevantes para cada comparación están incluidos en la Tabla 1.

\section{Discusión y Conclusiones}

Como se ha resumido previamente, hay cierta carencia de investigaciones sobre los métodos de enseñanza-aprendizaje de la expresividad emocional en la música y, particularmente, en el estudio de las concepciones sobre dichos métodos, de profesores y alumnos de niveles educativos superiores. Estas concepciones pueden influir en el modo en el que se realiza la práctica real en el aula y el presente trabajo representa una aportación empírica que trata de ofrecer información útil en este sentido, con el fin de ofrecer un instrumento que ayude a mejorar la práctica docente (y el entrenamiento de futuros docentes) mediante la reflexión de los resultados y conocer las concepciones sobre estrategias de aprendizaje de la expresividad musical.

El principal objetivo del trabajo era la validación de un instrumento de evaluación de las concepciones sobre los métodos usuales de enseñanza de la expresividad musical, los valores alfa de Cronbach fueron muy elevados para cada una de las cuatro subescalas (>.90 en todos los casos), especialmente teniendo en cuenta el escaso número de ítems en cada escala, 
lo que sugiere una excelente consistencia interna (De Vellis, 2003; Lance et al., 2006). Asimismo, el modelo factorial de las cuatro estrategias diferentes presentó un ajuste adecuado a los datos en el AFC (p.ej., $C F I=.948)$ y no hubo indicios de pesos factoriales inadecuados para ningún ítem en su escala teórica ni de cargas factoriales cruzadas. El pequeño tamaño de la muestra de profesores (para este tipo de análisis), sin embargo, no permitió realizar AFCs multigrupo para comprobar si la estructura era invariante entre ambos. En suma, los resultados permiten asumir que la estructura factorial del cuestionario es sólida y consistente.

Además, los factores latentes correlacionan todos entre ellos, lo que recalca la combinación de estrategias en la realidad del aula como sugieren algunos trabajos previos (Meissner, 2017). Además, las estrategias utilizadas en este trabajo son las más empleadas en la enseñanza de la expresividad, aunque algunos trabajos han sugerido que otras estrategias alternativas pueden ser útiles en la mejora de la expresividad. La posible mayor efectividad de estas y otras aproximaciones, sin embargo, puede no tener un impacto rápido y directo en la práctica en el aula y las estrategias estudiadas aquí, con elementos mezclados entre ellas y recogiendo ideas de otras perspectivas tiende a ser la experiencia cotidiana en las aulas de música.

Por otro lado, diferentes estudios indican que los movimientos y la gestualización del intérprete incrementan claramente la comprensión por parte de los oyentes de sus intenciones expresivas (Dahl y Friberg, 2007). Las estrategias propuestas están en relación plena con la expresión corporal y gestual, en línea con diferentes estudios que apoyan la idea de que el uso consciente del gesto y el movimiento expresivo puede ser una potente herramienta pedagógica en enseñanzas instrumentales (Massie-Laberge, Cosette y Wanderley, 2019). En las enseñanzas actuales el movimiento forma parte de la ejecución y es observable y experimentable en las cuatro estrategias propuestas.

En segundo lugar, las comparaciones entre ambos grupos indicaron que no existían diferencias entre ellos en el grado en el que habían estado en cada una de las situaciones descritas en el cuestionario. Los participantes habían vivenciado dichas situaciones, pero se apreciaba una diferencia entre grupos en las puntuaciones de la subescala centrada en el uso de metáforas o imágenes: los estudiantes puntuaban más alto que los profesores (aunque con un efecto medio: $g=0.37$ ). Es posible que los profesores, en mayor medida que los alumnos, valoren más los contras de esta estrategia relacionados con la percepción subjetiva de la información anteponiendo estos aspectos a los pros implicados. Como indican Juslin et al. 
(2006), las metáforas tienen el problema de que, aunque puedan ser muy evocadoras, suelen ser inevitablemente ambiguas, ya que pueden depender de la interpretación de cada alumno respecto a su experiencia personal particular. La legislación española en educación musical, igual que en otras áreas, ha ido progresivamente adoptando un enfoque en el que se valora especialmente la creatividad y la imaginación; podría considerarse que eso haya tenido algún efecto en la mejor valoración del modelo que más enfatiza este aspecto en el grupo de personas más jóvenes: los alumnos.

Más interesantes son las diferencias encontradas en los análisis intra-grupo sobre las puntuaciones totales en cada estrategia. Con pequeños matices, en ambos grupos se consideró por igual que la técnica era la más adecuada para la enseñanza de la expresividad, mientras que el modelado fue claramente la estrategia peor considerada. En consonancia con este resultado, se encontró que el uso de la técnica era la que tanto alumnos como profesores identificaban como más habitual en su experiencia docente o discente. La investigación previa ha sugerido, sin embargo, que el modelado es el método más usual en el aula de música (p.ej., Juslin et al., 2006), principalmente en niveles inferiores al analizado en este trabajo. Por tanto, esta diferencia puede ser una consecuencia del nivel educativo: en las enseñanzas superiores el nivel técnico y las destrezas alcanzadas por los alumnos son lógicamente más elevadas, y el aprendizaje y la ejecución expresiva de una pieza musical, requiere mayor precisión y consciencia del acto interpretativo. Por otro lado, hay datos que sugieren que la valoración del modelado es elevada cuando se considera que se aplica a niños pequeños (Bonastre y Timmers, 2019). De hecho, se conocen distintas formas de modelado y se considera que esta estrategia puede ser una herramienta útil dependiendo de cuándo, cómo y con quién se aplique, pero inadecuada en función de algunos factores como el contexto o tipos de alumnado (Haston, 2007).

Los resultados también indican que fue claramente el modelado la segunda estrategia más frecuente en la experiencia que informan ambos grupos, lo que no coincide con su valoración como el medio de enseñanza de la expresividad peor considerado. Los participantes en el estudio parece que creen que en estos niveles educativos el uso de esta estrategia es frecuente pero no efectivo. Su valoración negativa en comparación con las demás posibilidades planteadas puede reflejar un futuro cambio en el uso de estrategias y modelos educativos. El considerar que otras estrategias pueden aportar beneficios en el aprendizaje de la expresividad (aunque no consideren que su uso sea frecuente en el aula) puede ayudar a 
comenzar un cambio y una posible mejora respecto a actividades realizadas en la práctica habitual en la educación en niveles superiores. Esto puede tener particular relevancia ya que una parte importante de los actuales estudiantes serán futuros docentes en todos los niveles educativos.

Este trabajo presenta varias limitaciones. Sobre todo, como se comentaba anteriormente, el tamaño de una de las submuestras (los profesores) es algo pequeño. En todo caso, el total de la muestra se puede considerar una buena representación del grupo en sí, respecto al número de docentes implicados. Para los análisis realizados la muestra puede considerarse, además, suficiente aunque algunos efectos de pequeño tamaño han podido quedar parcialmente ocultos. Adicionalmente, el muestreo incidental y el recoger la muestra en un solo centro pueden limitar la generalizabilidad de los resultados. Aunque el RCSMM tiene un alumnado variado a nivel nacional e internacional, como se reflejaba anteriormente, hay que tomar con cautela la aplicación de los resultados de este trabajo a centros de otras comunidades o de otros países.

Las diferencias encontradas entre ambos grupos en las concepciones sobre la expresividad, específicamente en la mejor valoración por parte de los alumnos del uso de metáforas o imágenes podrían tenerse en cuenta en las planificaciones docentes respecto a la enseñanza de la expresividad. La preferencia de una u otra estrategia puede reflejar no sólo una valoración en sí misma sino también un uso determinado de los profesores en el aula (o de los futuros profesores) que puedan aportar una mejora expresiva real en el alumnado.

El instrumento de evaluación validado se propone como una herramienta útil en contextos educativos y de investigación sobre la expresividad en la música. Esta posible herramienta pedagógica puede aportar al aula una experiencia explícita y una posibilidad de discusión sobre la expresividad con los alumnos como punto de partida para una mejora real de los resultados en relación con la comunicación expresiva musical.

Por otro lado, aunque en el proceso de enseñanza-aprendizaje los resultados indican que las estrategias usadas aquí son relevantes en la práctica actual hay que considerar que nuevas líneas metodológicas pueden ampliar y mejorar el uso de estas estrategias como el método dialógico que es un tipo de estrategia instruccional basada en el diálogo entre el alumno y el profesor en lugar de estar centrado en las instrucciones unidireccionales 
(Karlsson, 2008); parece que este método ha funcionado bien en varios trabajos recientes con niños (Meissner y Timmers, 2020) y es totalmente compatible con las estrategias propuestas añadiendo retroalimentación en la acción pedagógica. Asimismo, está demostrando ser útil el uso de distintos sistemas de representación para reconstruir la propia experiencia musical (Pozo, Torrado y Pérez-Echeverría, 2019), lo cual puede ser compatible con las estrategias planteadas.

Futuros trabajos podrían analizar en mayor profundidad la validez de este instrumento de evaluación, así como estudiar el efecto que el rol docente vs. discente puede tener tanto en las concepciones de ambos grupos como su efecto en el proceso educativo. Para ello, contar con estudios longitudinales y trabajos cualitativos sería un aporte que nos permitiría definir mejor qué se hace, para qué y cómo mejorarlo. Estudiar la relación de las estrategias evaluadas mediante el cuestionario que se valida en este estudio, las que tradicionalmente se usan con más frecuencia, con las alternativas que se van proponiendo recientemente para la mejora de la expresividad en la interpretación musical, sería de gran interés con el fin de optimizar las herramientas pedagógicas disponibles y facilitar una mayor consciencia y sensibilización en la comunidad educativa hacia este aspecto completamente central en la música y, por tanto, en la formación de los músicos de todos los niveles.

\section{Referencias}

Bonastre, C., Muñoz, E. y Timmers; R. (2017). Conceptions about teaching and learning of expressivity in music among Higher Education teachers and students. British Journal of Music Education, 34, 277-290. doi: 10.1017/S0265051716000462

Bonastre, C. y Timmers, R. (2019). Comparison of beliefs about teaching and learning of emotional expression in music performance between Spanish and English HE students of music. Psychology of Music,1-16. doi: 10.1177/0305735619842366

Bautista, A. y Pérez-Echeverría, M.P. (2008). ¿Qué consideran los profesores de instrumento que deben enseñar en sus clases? Cultura y Educación, 20, 17-34. doi: $10.1174 / 113564008783781477$.

Bentler, P.M. (1990). Comparative fit indexes in structural models. Psychological Bulletin, 107, 238-246. doi: 10.1037/0033-2909.107.2.238. 
Brenner, B. y Strand, K. (2013). A case study of teaching musical expression to young performers. Journal of Research in Music Education, 61, 80-96. doi: 10.1177/0022429412474826.

Broomhead, P. (2006). A Study of Instructional Strategies for Teaching Expressive Performance in the Choral Rehearsal. Bulletin of the Council for Research in Music Education, 167, 7-20. https://www.jstor.org/stable/40319286.

Casas-Mas, A., Pozo, J.I. y Montero, I. (2014). The influence of musical learning cultures on the construction of teaching-learning conceptions. British Journal of Music Education, 31, 319-342. doi: 10.1017/S0265051714000096.

Chaffin, R. y Lemieux, A.F. (2005). Consideraciones generales sobre el perfeccionamiento musical . Quodlibet, 32, 22-48.

Cohen, J. (1988). Statistical power analysis for the behavioral sciences. Hillsdale: NJ: Erlbaum (2nd ed.).

Dahl, S. y Friberg, A. (2007). Visual perception of expressiveness in musicians' body movements. Music Perception, 24, 433-454. doi: 10.1525/mp.2007.24.5.433.

De Vellis, R. (2003). Scale development: theory and applications: theory and application. Thousand Okas, CA: Sage.

Doğantan-Dack, M. (2014). Philosophical reflections on expressive music performance. En D. Fabian, R. Timmers y E. Schubert. (Eds.) Expressiveness in music performance. Empirical approaches across styles and cultures (pp. 3-21). Oxford: Oxford University Press.

Fang, Z. (1996). A review of research on teacher beliefs and practices. Educational Research, 38, 47-65. doi: 10.1080/0013188960380104.

Gabrielsson, A. y Juslin, P.N. (2003). Emotional expression in music'. En R.J. Davidson, H. H. Goldsmith y K. R. Scherer (Eds.), Handbook of affective sciences (pp. 503-534). New York: Oxford University Press.

Garnett, J. (2013). Beyond a constructivist curriculum: a critique of competing paradigms in music education. British Journal of Music Education, 30, 161-175. doi: $10.1017 / \mathrm{S} 0265051712000575$.

González Rollo, A. y Bautista, A. (2018). Ideas de los profesores de instrumento en conservatorios sobre cómo calificar: aspectos objetivos y subjetivos de la interpretación musical. Publicaciones, 48, 127-148. doi: 10.30827/publicaciones.v4812.8337. 
Hallam, S. (2010). Music education. The role of affect. En P. N. Juslin y J. A. Sloboda (Eds), Handbook of Music and Emotion: Theory, Research, Applications (pp. 791-817). Oxford: Oxford University Press.

Haston, W. (2007). Teacher modeling as an effective teaching strategy. Music Educators Journal, 93, 26-30. doi: 10.1177/002743210709300414.

Hughes, R. y Huby, M. (2004). The construction and interpretation of vignettes in social research. Social Work and Social Sciences Review, 11, 36-51. doi: 10.1921/17466105.11.1.36.

Juslin, P.N., Karlsson, J., Lindström, E.F., Friberg, A. y Schoonderwaldt, E. (2006). Play it again with feeling: Computer feedback in musical communication of emotions. Journal of Experimental Psychology: Applied, 12, 79-95. doi: 10.1037/1076-898X.12.2.79.

Juslin, P.N. y Persson, R.S. (2002). The science and psychology of music performance: Creative strategies for teaching and learning. En R. Parncutt y G.E. McPherson (Eds.), Emotional communication (pp. 219-236). New York: Oxford University Press.

Karlsson, J. y Juslin, P.N. (2008). Musical expression: An observational study of instrumental teaching. Psychology of Music, 36, 309-334. doi: 10.1177/0305735607086040.

Lance, C.E., Butts, M.M. y Michels, L.C. (2006). The Sources of Four Commonly Reported Cutoff Criteria: What Did They Really Say? Organizational Research Methods, 9, 20220. doi: $10.1177 / 1094428105284919$.

López-Iñiguez, G., Pozo J. y de Dios M.J. (2014). The older, the wiser? Profiles of string instrument teachers with different experience according to their conceptions of teaching, learning, and evaluation. Psychology of Music, 41, 1-20. doi: $10.1177 / 0305735612463772$.

MacCallum, R.C., Widaman, K.F., Zhang, S., y Hong, S. (1999). Sample size in factor analysis. Psychological Methods, 4, 84-99. doi: 10.1037/1082-989X.4.1.84.

Marchand, D.J. (1975). A study of two approaches to developing expressive performance. Journal of Research in Music Education, 23, 14-22. doi: 10.2307/3345199.

Massie-Laberge, C., Cosette, I. y Wanderley, M.M. (2019). Kinematic Analysis of Pianists' Expressive Performances of Romantic Excerpts: Applications for Enhanced Pedagogical Approaches. Frontiers in Psychology, 9:2725. doi: 10.3389/fpsyg.2018.02725.

Meissner, H. (2017). Instrumental teachers' instructional strategies for facilitating children's learning of expressive music performance: An exploratory study. International Journal of Music Education, 35, 118-135. doi: 10.1177/0255761416643850. 
Meissner, H. y Timmers, R. (2019). Teaching young musicians expressive performance: An experimental study. Music Education Research., 21, 20-39. doi: 10.1080/14613808.2018.1465031.

Meissner, H., Timmers, R.. (2020). Young musicians' learning of expressive performance: the Importance of dialogic teaching and modeling. Frontiers in Education, 5:11. doi: 10.3389/feduc.2020.00011.

Pozo, J.I., Torrado, J.A. y Pérez-Echevarría, M.P. (2019). Aprendiendo a interpretar música por medio del Smartphonela explicitación y reconstrucción de las representaciones encarnadas. Cuadernos del Centro de Estudios en Diseño y Comunicación. Ensayos, 89, 237-260. Artículo recuperado el 20 de maezo de 2020 de: http://fido.palermo.edu/servicios_dyc/publicacionesdc/archivos/783_libro.pdf.

Sánchez, G. y López, M. (2019). Análisis de los contenidos de expresión corporal impartidos en la formación inicial de los docentes de primaria. Educación XXI, 22, 425-447. doi: 10.5944/educXX1.20058.

Sloboda, J.A., Gayford, C. y Minassian, C. (2003). Assisting advanced musicians to enhance their expressivity: An intervention study. Communication presented at the Fifth Triennial Conference of the European Society for the Cognitive Sciences of Music, Hannover, Septiembre.

Torrado, J.A. y Pozo, J.I. (2008). Metas y estrategias para una práctica constructiva en la enseñanza de la música. Cultura y Educación, 20, 35-48. doi: $10.1174 / 113564008783781468$.

Viellard, S., Roy, M. y Peretz, I. (2012). Expressivenes in musical emotions. Psychological Research, 76, 641-653. doi: 10.100s0042601103614.

Williamon, A. (2014). Implications for education. En D. Fabian, R. Timmers y E. Schubert, Expressiveness in music performance. Empirical approaches across styles and cultures (pp.348-352). Oxford: Oxford University Press.

Woody, R. H. (2006). The effect of various instructional conditions on expressive music performance. Journal of Research and Music Education, 54, 21-36. doi: $10.1177 / 002242940605400103$.

Young, V,. Burwell, K. y Pickup, D. (2003). Areas of study and teaching strategies in instrumental teaching: A case study research project. Music Education Research, 5, 139155. doi: 10.1080/1461380032000085522. 
Recibido: 31-10-2019

Aceptado: $19-04-2020$ 